\title{
Efficient Verification of Network Fault Tolerance via Counterexample-Guided Refinement
}

\author{
Nick Giannarakis ${ }^{1(\bowtie)}$, Ryan Beckett ${ }^{2}$, \\ Ratul Mahajan ${ }^{3,4}$, and David Walker ${ }^{1}$ \\ 1 Princeton University, Princeton, NJ 08544, USA \\ $\{$ ng8, dpw $@$ cs . princeton. edu \\ 2 Microsoft Research, Redmond, WA 98052, USA \\ ryan.beckett@microsoft.com \\ 3 University of Washington, Seattle, WA 98195, USA \\ ratul@cs. washington. edu \\ 4 Intentionet, Seattle, WA, USA
}

\begin{abstract}
We show how to verify that large data center networks satisfy key properties such as all-pairs reachability under a bounded number of faults. To scale the analysis, we develop algorithms that identify network symmetries and compute small abstract networks from large concrete ones. Using counter-example guided abstraction refinement, we successively refine the computed abstractions until the given property may be verified. The soundness of our approach relies on a novel notion of network approximation: routing paths in the concrete network are not precisely simulated by those in the abstract network but are guaranteed to be "at least as good." We implement our algorithms in a tool called Origami and use them to verify reachability under faults for standard data center topologies. We find that Origami computes abstract networks with 1-3 orders of magnitude fewer edges, which makes it possible to verify large networks that are out of reach of existing techniques.
\end{abstract}

\section{Introduction}

Most networks decide how to route packets from point A to B by executing one or more distributed routing protocols such as the Border Gateway Protocol (BGP) and Open Shortest Path First (OSPF). To achieve end-to-end policy objectives related to cost, load balancing, security, etc., network operators author configurations for each router. These configurations control various aspects of the route computation such as filtering and ranking route information received from neighbors, information injection from one protocol to another, and so on.

This work was supported in part by NSF Grants 1703493 and 1837030, and gifts from Cisco and Facebook. Any opinions, findings, and conclusions expressed are those of the authors and do not necessarily reflect those of the NSF, Cisco or Facebook.

(C) The Author(s) 2019

I. Dillig and S. Tasiran (Eds.): CAV 2019, LNCS 11562, pp. 305-323, 2019.

https://doi.org/10.1007/978-3-030-25543-5_18 
This flexibility, however, comes at a cost: Configuring individual routers to enforce the desired policies of the distributed system is complex and errorprone $[15,21]$. The problem of configuration is further compounded by three challenges. The first is network scale. Large networks such as those of cloud providers can consist of millions of lines of configuration spread across thousands of devices. The second is that operators must account for the interaction with external neighbors who may sent arbitrary routing messages. Finally one has to deal with failures. Hardware failures are common [14] and lead to a combinatorial explosion of different possible network behaviors.

To combat the complexity of distributed routing configurations, researchers have suggested a wide range of network verification $[2,13,25]$ and simulation $[11,12,23]$ techniques. These techniques are effective on small and medium-sized networks, but they cannot analyze data centers with $1000 \mathrm{~s}$ of routers and all their possible failures. To enable scalable analyses, it seems necessary to exploit the symmetries that exist in most large real networks. Indeed, other researchers have exploited symmetries to scale verification in the past $[3,22]$. However, it has never been possible to account for failures, as they introduce asymmetries that change routing behaviors in unpredictable ways.

To address this challenge, we develop a new algorithm for verifying reachability in networks in the presence of faults, based on the idea of counterexampleguided abstraction refinement (CEGAR) [5]. The algorithm starts by factoring out symmetries using techniques developed in prior work [3] and then attempts verification of the abstract network using an SMT solver. If verification succeeds, we are done. However, if verification fails, we examine the counter-example to decide whether we have a true failure or we must refine the network further and attempt verification anew. By focusing on reachability, the refinement procedure can be accelerated by using efficient graph algorithms, such as min cut, to rule out invalid abstractions in the middle of the CEGAR loop.

We prove the correctness of our algorithm using a new theory of faulty networks that accounts for the impact of all combinations of $k$ failures. Our key insight is that, while routes computed in the abstract network may not simulate those of the concrete network exactly, under the right conditions they are guaranteed to approximate them. The approximation relation between concrete and abstract networks suffices to verify key properties such as reachability.

We implemented our algorithms in a tool called Origami and measured their performance on common data center network topologies. We find that Origami computes abstract networks with 1-3 orders of magnitude fewer edges. This reduction speeds verification dramatically and enables verification of networks that are out of reach of current state-of-the-art tools [2].

\section{Key Ideas}

The goal of Origami is to speed up network verification in the presence of faults, and it does so by computing small, abstract networks with similar behavior to a given concrete network. 


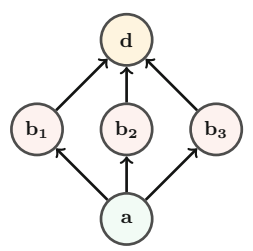

(a)

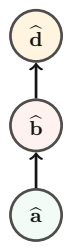

(b)

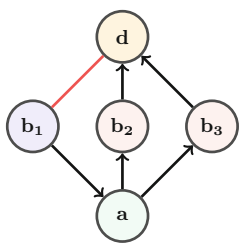

(c)

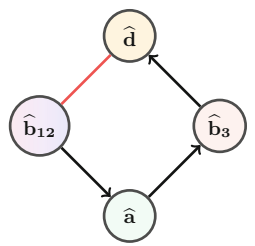

(d)

Fig. 1. All graph edges shown correspond to edges in the network topology, and we draw edges as directed to denote the direction of forwarding eventually determined for each node by the distributed routing protocols for a fixed destination $d$. In (a) nodes use shortest path routing to route to the destination $d$. (b) shows a compressed network that precisely captures the forwarding behavior of (a). (c) shows how forwarding is impacted by a link failure, shown as a red line. (d) shows a compressed network that is sound approximation of the original network for any single link failure. (Color figure online)

As a first approximation, one can view a network as a directed graph capturing the physical topology, and its routing solution as a subgraph where the remaining edges denote the forwarding decision at each node for some fixed destination. In the absence of faults, given a concrete and abstract network, one can define a natural notion of similarity as a graph homomorphism: assigning each concrete node a corresponding abstract node such that, for any solution to the routing problem, the concrete node forwards "in the same direction" as the corresponding abstract node. For example, the concrete network in Fig. 1a is related to its abstract counterpart in Fig. $1 \mathrm{~b}$ according to the node colors.

Unfortunately, we run into two significant problems when defining abstractions in this manner in the presence of faults. First, the concrete nodes of Fig. 1a have at least 2 disjoint paths to the destination whereas abstract nodes of Fig. $1 \mathrm{~b}$ have just one path to the destination, so the abstract network does not preserve the desired fault tolerance properties. Second, consider Fig. 1c, which illustrates how the routing decisions change when a failure occurs. Here, the nodes $\left(b_{1}\right.$ in particular) no longer route "in the same direction" as the original network or its abstraction. Hence the invariant connecting concrete and abstract networks is violated.

Lossy Compression. To achieve compression given a bounded number of link failures, we relax the notion of similarity between concrete and abstract nodes: A node in the abstract network may merely approximate the behavior of concrete nodes. This makes it possible to compress nodes that, in the presence of failures, may route differently. In general, when we fail a single link in the abstract network, we are over-approximating the failures in the concrete network by failing multiple concrete links, possibly more than desired. Nevertheless, the paths taken in the concrete network can only deviate so much from the paths found in the abstract network: 
Property 1. If a node has a route to the destination in the presence of $k$ link failures then it has a route that is "at least as good" (as prescribed by the routing protocol) in the presence of $k^{\prime}$ link failures for $k^{\prime}<k$.

This relation suffices to verify important network reliability properties, such as reachability, in the presence of faults. Just as importantly, it allows us to achieve effective network compression to scale verification.

Revisiting our example, consider the new abstract network of Fig. 1d. When the link between $\widehat{b}_{12}$ and $\widehat{d}$ has failed, $\widehat{b}_{12}$ still captures the behavior of $b_{1}$ precisely. However, $b_{2}$ has a better (in this case better means shorter) path to $d$. Despite this difference, if the operator's goal was to prove reachability to the destination under any single fault, then this abstract network suffices.

From Specification to Algorithm. It is not too difficult to find abstract networks that approximate a concrete network; the challenge is finding a valid abstract network that is small enough to make verification feasible and yet large enough to include sufficiently many paths to verify the given fault tolerance property. Rather than attempting to compute a single abstract network with the right properties all in one shot, we search the space of abstract networks using an algorithm based on counter-example guided abstraction refinement [5].

The CEGAR algorithm begins by computing the smallest possible valid abstract network. In the example above, this corresponds to the original compressed network in Fig. 1b, which faithfully approximates the original network when there are no link failures. However, if we try to verify reachability in the presence of a single fault, we will conclude that nodes $\widehat{b}$ and $\widehat{a}$ have no route to the destination when the link between $\widehat{b}$ and $\widehat{d}$ fails. The counterexample due to this failure could of course be spurious (and indeed it is). Fortunately, we can easily distinguish whether such a failure is due to lack of connectivity or an artifact of over-abstracting, by calculating the number of corresponding concrete failures. In this example a failure on the link $\langle\widehat{b}, \widehat{d}\rangle$ corresponds to 3 concrete failures. Since we are interested in verifying reachability for a single failure this cannot constitute an actual counterexample.

The next step is to refine our abstraction by splitting some of the abstract nodes. The idea is to use the counterexample from the previous iteration to split the abstract network in a way that avoids giving rise to the same spurious counterexample in the next iteration (Sect. 5). Doing so results in the somewhat larger network of Fig.1d. A second verification pass over this larger network takes longer, but succeeds.

\section{The Network Model}

Though there are a wide variety of routing protocols in use today, they share a lot in common. Griffin et al. [16] showed that protocols like BGP and others solve instances of the stable paths problem, a generalization of the shortest paths problem, and Sobrinho [24] demonstrated their semantics and properties can be 
modelled using routing algebras. We extend these foundations by defining stable paths problems with faults (SPPFs), an extension of the classic Stable Paths Problem that admits the possibility of a bounded number of link failures. In later sections, we use this network model to develop generic network compression algorithms and reason about their correctness.

Stable Path Problems with Faults (SPPFs): An SPPF is an instance of the stable paths problem with faults. Informally, each instance defines the routing behavior of an operational network. The definition includes both the network topology as well as the routing policy. The policy specifies the way routing messages are transformed as they travel along links and through the user-configured import and export filters/transformers of the devices, and also how the preferred routes are chosen at a given device. In our formulation, each problem instance also incorporates a specification of the possible failures and their impact on the routing solutions.

Formally, an SPPF is a tuple with six components:

1. A graph $G=\langle V, E\rangle$ denoting the network topology.

2. A set of "attributes" (i.e., routing messages) $A_{\infty}=A \cup\{\infty\}$ that may be exchanged between devices. The symbol $\infty$ represents the absence of a route.

3. A destination $d \in V$ and its initial route announcement $a_{d} \in A$. For simplicity, each SPPF has exactly one destination $(d)$. (To model a network with many destinations, one would use a set of SPPFs.)

4. A partial order $\prec \subseteq A_{\infty} \times A_{\infty}$ ranks attributes. If $a \prec b$ then we say route $a$ is preferred over route $b$. Any route $a \in A$ is preferred to no route $(a \prec \infty)$.

5. A function trans : $E \rightarrow A_{\infty} \rightarrow A_{\infty}$ that denotes how messages are processed across edges. This function models the route maps and filters that transform route announcements as they enter or leave routers.

6. A bound $k$ on the maximum number of link failures that may occur.

Examples: By choosing an appropriate set of routing attributes, a preference relation and a transfer function, one can model the semantics of commonly used routing protocols. For instance, the Routing Information Protocol (RIP) is a simple shortest paths protocol. It can be modelled by an SPPF where (1) the set of attributes $A$ is the set of integers between 0 and 15 (i.e., the set of permitted path lengths), (2) the preference relation is integer inequality so shorter paths are preferred, and (3) the transfer function increments the received attribute by 1 or drops the route if it exceeds the maximum hop count of 15 :

$$
\operatorname{trans}(e, a)= \begin{cases}\infty & \text { if } a \geq 15 \\ a+1 & \text { otherwise }\end{cases}
$$

Going beyond simple shortest paths, BGP is a complex, policy-driven protocol that drives the Internet, and increasingly, data centers [18]. Operators often choose BGP due to its high expressiveness. We can model a version of BGP (simplified for presentation) using messages consisting of triples (LP, Comm, Path) 
where LP is an integer-valued local preference, Comm is a set of community values (which are essentially string tags) and Path is a list of nodes, representing the path a routing message has traversed. The transfer function always adds the current device to the Path (or drops the message if a loop is detected) and will modify the LP and Comm components of the attribute according to the device configuration. For instance, one device may attach a community tag to a route and another device may filter or modify routes that have the tag attached. The protocol semantics dictates the preference relation (preferring routes with higher local preference first, and shorter paths second). A more complete BGP model is not fundamentally harder to model - it simply has additional attribute fields and more complex transfer and preference relations [20].

SPPF Solutions: In a network, routers will repeatedly exchange messages, applying their transfer functions to neighbor routes and selecting a current best route based on the preference relation, until the network reaches a fixpoint (stable state). Interestingly, Griffin et al. [16] showed that all routing solutions can be described via a set of local stability constraints. We exploit this insight to define a series of logical constraints that capture all possible routing behaviors in a setting that includes link failures. More specifically, we define a solution (aka, stable state) $\mathcal{S}$ of an SPPF to be a pair $\langle\mathcal{L}, \mathcal{F}\rangle$ of a labelling $\mathcal{L}$ and a failure scenario $\mathcal{F}$. The labelling $\mathcal{L}$ is an assignment of the final attributes to nodes in the network. If an attribute $a$ is assigned to node $v$, we say that node has selected (or prefers) that attribute over other attributes available to it. The chosen route also determines packet forwarding. If a node $X$ selects a route from neighbor $Y$, then $X$ will forward packets to $Y$. The failure scenario $\mathcal{F}$ is an assignment of 0 (has not failed) or 1 (has failed) to each edge in the network.

A solution $\mathcal{S}=\langle\mathcal{L}, F\rangle$ to an $\operatorname{SPPF}=\left(G, A, a_{\mathrm{d}}, \prec\right.$, trans, $\left.k\right)$ is a stable state satisfying the following conditions:

$$
\begin{aligned}
& \mathcal{L}(u)= \begin{cases}a_{\mathrm{d}} & u=d \\
\infty & \operatorname{choices}_{\mathcal{S}}(u)=\emptyset \\
\min _{\prec}\left(\left\{a \mid(e, a) \in \operatorname{choices}_{\mathcal{S}}(u)\right\}\right) & \operatorname{choices}_{\mathcal{S}}(u) \neq \emptyset\end{cases} \\
& \text { subject to } \sum_{e \in E} \mathcal{F}(e) \leq k
\end{aligned}
$$

where the choices from the neighbors of node $u$ are defined as:

$$
\operatorname{choices}_{\mathcal{S}}(u)=\{(e, a) \mid e=\langle u, v\rangle, a=\operatorname{trans}(e, \mathcal{L}(v)), a \neq \infty, \mathcal{F}(e)=0\}
$$

The constraints require that every node has selected the best attribute (according to its preference relation) amongst those available from its neighbors. The destination's label must always be the initial attribute $a_{d}$. For verification, this attribute (or parts of it) may be symbolic, which helps model potentially unknown routing announcements from peers outside our network. For other nodes $u$, the selected attribute $a$ is the minimal attribute from the choices available to $u$. Intuitively, to find the choices available to $u$, we consider 
the attributes $b$ chosen by neighbors $v$ of $u$. Then, if the edge between $v$ and $u$ is not failed, we push $b$ along that edge, modifying it according to the trans function. Finally, failure scenarios are constrained so that the sum of the failures is at most $k$.

\section{Network Approximation Theory}

Given a concrete SPPF and an abstract $\widehat{\text { SPPF }}$, a network abstraction is a pair of functions $(f, h)$ that relate the two. The topology abstraction $f: V \rightarrow \widehat{V}$ maps each node in the concrete network to a node in the abstract network, while the attribute abstraction $h: A_{\infty} \rightarrow \widehat{A}_{\infty}$ maps a concrete attribute to an abstract attribute. The latter allows us to relate networks running protocols where nodes may appear in the attributes (e.g. as in the Path component of BGP).

The goal of Origami is to compute compact $\widehat{\text { SPPFs }}$ that may be used for

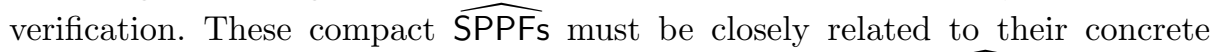
counterparts. Otherwise, properties verified on the compact $\widehat{\text { SPPF }}$ will not be true of their concrete counterpart. Section 4.1 defines label approximation, which provides an intuitive, high-level, semantic relationship between abstract and concrete networks. We also explain some of the consequences of this definition and its limitations. Unfortunately, while this broad definition serves as an important theoretical objective, it is difficult to use directly in an efficient algorithm. Section 4.2 continues our development by explaining two well-formedness requirements of network policies that play a key role in establishing label approximation indirectly. Finally, Sect. 4.3 defines effective SPPF approximation for well-formed SPPFs. This definition is more conservative than label approximation, but has the advantage that it is easier to work with algorithmically and, moreover, it implies label approximation.

\subsection{Label Approximation}

Intuitively, we say the abstract $\widehat{\text { SPPF }}$ label-approximates the concrete SPPF when SPPF has at least as good a route at every node as $\widehat{\text { SPPF }}$ does.

Definition 1 (Label Approximation). Consider any solutions $\mathcal{S}$ to SPPF and $\widehat{\mathcal{S}}$ to $\widehat{\mathrm{SPPF}}$ and their respective labelling components $\mathcal{L}$ and $\widehat{\mathcal{L}}$. We say $\widehat{\mathrm{SPPF}}$ label-approximates SPPF when $\forall u \in V . h(\mathcal{L}(u)) \preceq \widehat{\mathcal{L}}(f(u))$.

If we can establish a label approximation relation between a concrete and an abstract network, we can typically verify a number of properties of the abstract network and be sure they hold of the concrete network. However, the details of exactly which properties we can verify depend on the specifics of the preference relation $(\prec)$. For example, in an OSPF network, preference is determined by weighted path length. Therefore, if we know an abstract node has a path of weighted length $n$, we know that its concrete counterparts have paths of weighted length of at most $n$. More importantly, since "no route" is the worst route, we 
know that if a node has any route to the destination in the abstract network, so do its concrete counterparts.

Limitations. Some properties are beyond the scope of our tool (independent of the preference relation). For example, our model cannot reason about quantitative properties such as bandwidth, probability of congestion, or latency.

\subsection{Well-Formed SPPFs}

Not all SPPFs are well-behaved. For example, some never converge and others do not provide sensible models of any real network. To avoid dealing with such poorly-behaved models, we demand henceforth that all SPPFs are wellformed. Well-formedness entails that an SPPF is strictly monotonic and isotonic: $\forall a, e . \quad a \neq \infty \Rightarrow a \prec \operatorname{trans}(e, a)$ $\forall a, b, e . \quad a \preceq b \Rightarrow \operatorname{trans}(e, a) \preceq \operatorname{trans}(e, b)$ strict monotonicity
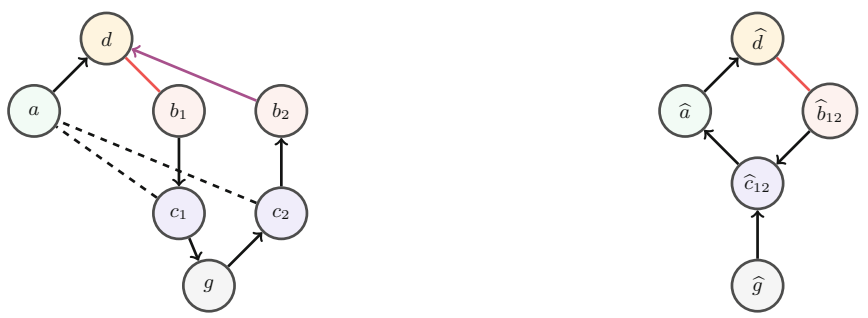

Fig. 2. Concrete network (left) and its corresponding abstraction (right). Nodes $c_{1}, c_{2}$ prefer to route through $b_{1}$ (resp. $b_{2}$ ), or $g$ over $a$. Node $b_{1}$ (resp. $b_{2}$ ) drops routing messages that have traversed $b_{2}$ (resp. $b_{1}$ ). Red lines indicate a failed link. Dotted lines show a topologically available but unused link. A purple arrow show a route unusable by traffic from $b_{1}$. (Color figure online)

Monotonicity and isotonicity properties are often cited $[7,8]$ as desirable properties of routing policies because they guarantee network convergence and prevent persistent oscillation. In practice too, prior studies have revealed that almost all real network configurations have these properties [13,19].

In our case, these properties help establish additional invariants that tie the routing behavior of concrete and abstract networks together. To gain some intuition as to why, consider the networks of Fig. 2. The concrete network on the left runs BGP with the routing policy that node $c_{1}$ (and $c_{2}$ ) prefers to route through node $g$ instead of $a$, and that $b_{1}$ drops announcements coming from $b_{2}$. In this scenario, the similarly configured abstract node $\widehat{b}_{12}$ can reach the destination - it simply takes a route that happens to be less preferred by $\hat{c}_{12}$ than it would if there had been no failure. However, in the concrete analogue, $b_{1}$, is unable to reach the destination because $c_{1}$ only sends it the route through $b_{2}$, which it cannot use. In this case, the concrete network has more topological 
paths than the abstract network, but, counterintuitively, due to the network's routing policy, this turns out to be a disadvantage. Hence having more paths does not necessarily make nodes more accessible. As a consequence, in general, abstract networks cannot soundly overapproximate the number of failures in a concrete network - an important property for the soundness of our theory.

The underlying issue here is that the networks of Fig. 2 are not isotonic: suppose $\mathcal{L}^{\prime}\left(c_{1}\right)$ is the route from $c_{1}$ to the destination through node $a$, we have that $\mathcal{L}\left(c_{1}\right) \prec \mathcal{L}^{\prime}\left(c_{1}\right)$ but since the transfer function over $\left\langle b_{1}, c_{1}\right\rangle$ drops routes that have traversed node $b_{2}$, we have that $\operatorname{trans}\left(\left\langle b_{1}, c_{1}\right\rangle, \mathcal{L}\left(c_{1}\right)\right) \nprec \operatorname{trans}\left(\left\langle b_{1}, c_{1}\right\rangle, \mathcal{L}^{\prime}\left(c_{1}\right)\right)$. Notice that $\mathcal{L}^{\prime}\left(c_{1}\right)$ is essentially the route that the abstract network uses i.e. $h\left(\mathcal{L}^{\prime}\left(c_{1}\right)\right)=\widehat{\mathcal{L}}\left(\hat{c}_{12}\right)$, hence the formula above implies that $h\left(\mathcal{L}\left(b_{1}\right)\right) \nprec \widehat{\mathcal{L}}\left(\hat{b}_{12}\right)$ which violates the notion of label approximation. Fortunately, if a network is strictly monotonic and isotonic, such situations never arise. Moreover, we check these properties via an SMT solver using a local and efficient test.

\subsection{Effective SPPF Approximation}

We seek abstract networks that label-approximate given concrete networks. Unfortunately, to directly check that a particular abstract network label approximates a concrete network one must effectively compute their solutions. Doing so would defeat the entire purpose of abstraction, which seeks to analyze large concrete networks without the expense of computing their solutions directly.

In order to turn approximation into a useful computational tool, we define effective approximation, a set of simple conditions on the abstraction functions $f$ and $h$ that are local and can be checked efficiently. When true those conditions imply label approximation. Intuitively effective approximations impose three main restrictions on the abstraction functions:

1. The topology abstraction conforms to the $\forall \exists$-abstraction condition; this requires that there is an abstract edge $(\widehat{u}, \widehat{v})$ iff for every concrete node $u$ such that $f(u)=\widehat{u}$ there is some node $v$ such that $f(v)=\widehat{v}$ and $(u, v) \in E$.

2. The abstraction preserves the rank of attributes (rank-equivalence):

$$
\forall a, b . a \prec b \Longleftrightarrow h(a) \prec h(b)
$$

3. The transfer function and the abstraction functions commute (transequivalence):

$$
\forall e, a \cdot h(\operatorname{trans}(e, a))=\widehat{\operatorname{trans}}(f(e), h(a))
$$

We prove that when these conditions hold, we can approximate any solution of the concrete network with a solution of the abstract network.

Theorem 1. Given a well-formed SPPF and its effective approximation $\widehat{\mathrm{SPPF}}$, for any solution $\mathcal{S} \in S P P F$ there exists a solution $\widehat{\mathcal{S}} \in \widehat{\mathrm{SPPF}}$, such that their labelling functions are label approximate. 


\section{The Verification Procedure}

The first step of verification is to compute a small abstract network that satisfies our SPPF effective approximation conditions. We do so by grouping network nodes and edges with equivalent policy and checking the forall-exists topological condition, using an algorithm reminiscent of earlier work [3]. Typically, however, this minimal abstraction will not contain enough paths to prove any faulttolerance property. To identify a finer abstraction for which we can prove a fault-tolerance property we repeatedly:

1. Search the set of candidate refinements for the smallest plausible abstraction.

2. If the candidate abstraction satisfies the desired property, terminate the procedure. (We have successfully verified our concrete network.)

3. If not, examine whether the returned counterexample is an actual counterexample. We do so, by computing the number of concrete failures and check that it does not exceed the desired bound of link failures. (If so, we have found a property violation.)

4. If not, use the counterexample to learn how to expand the abstract network into a larger abstraction and repeat.

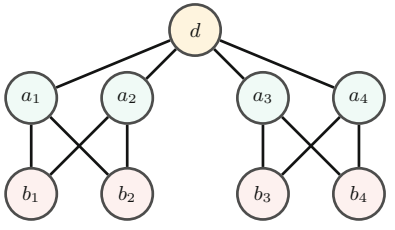

(a)

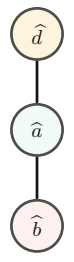

(b)

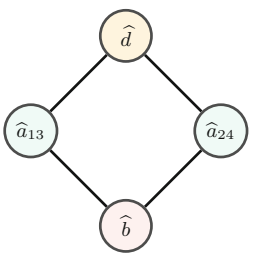

(c)

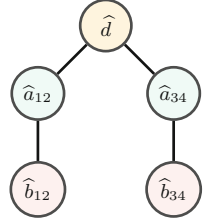

(d)

Fig. 3. Eight nodes in (a) are represented using two nodes in the abstract network (b). Pictures (c) and (d) show two possible ways to refine the abstract network (b).

Both the search for plausible candidates and the way we learn a new abstraction to continue the counterexample-guided loop are explained below.

\subsection{Searching for Plausible Candidates}

Though we might know an abstraction is not sufficient to verify a given fault tolerance property, there are many possible refinements. Consider, for example, Fig. 3(a) presents a simple concrete network that will tolerate a single link failure, and Fig. 3(b) presents an initial abstraction. The initial abstraction will not tolerate any link failure, so we must refine the network. To do so, we choose an abstract node to divide into two abstract nodes for the next iteration. We must also decide which concrete nodes correspond to each abstract node. For example, in Fig. 3(c), node $\hat{a}$ has been split into $\hat{a}_{13}$ and $\hat{a}_{24}$. The subscripts indicate the assignment of concrete nodes to abstract ones. 
A significant complication is that once we have generated a new abstraction, we must check that it continues to satisfy the effective approximation conditions, and if not, we must do more work. Figure $3(\mathrm{c})$ satisfies those conditions, but if we were to split $\hat{a}$ into $\hat{a}_{12}$ and $\hat{a}_{34}$ rather than $\hat{a}_{13}$ and $\hat{a}_{24}$, the forall-exists condition would be violated - some of the concrete nodes associated with $\hat{b}$ are connected to the concrete nodes in $\hat{a}_{12}$ but not to the ones in $\hat{a}_{34}$ and vice versa. To repair the violation of the forall-exists condition, we need to split additional nodes. In this case, the $\hat{b}$ node, giving rise to diagram Fig. 3(d).

Overall, the process of splitting nodes and then recursively splitting further nodes to repair the forall-exists condition generates many possible candidate abstractions to consider. A key question is which candidate should we select to proceed with the abstraction refinement algorithm?

One consideration is size: A smaller abstraction avoids taxing the verifier, which is the ultimate goal. However, there are many small abstractions that we can quickly dismiss. Technically, we say an abstraction is plausible if all nodes of interest have at least $k+1$ paths to the destination. Implausible abstractions cause nodes to become unreachable with $k$ failures. To check whether an abstraction is plausible, we compute the min-cut of the graph. Figure 3(d) is an example of an implausible abstraction that arose after a poorly-chosen split of node $\hat{a}$. In this case, no node has 2 or more paths to the destination and hence they might not be able to reach the destination when there is a failure.

Clearly verification using an implausible abstraction will fail. Instead of considering such abstractions as candidates for running verification on, the refinement algorithm tries refining them further. A key decision the algorithm needs to make when refining an abstraction is which abstract node to split. For instance, the optimal refinement of Fig. 3(b) is Fig. 3(c). If we were to split node $\hat{b}$ instead of $\hat{a}$ we would end up with a sub-optimal (in terms of size) abstraction. Intuitively, splitting a node that lies on the min-cut and can reach the destination (e.g. $\hat{a}$ ) will increase the number of paths that its neighbors on the unreachable part of the min-cut (e.g. $\hat{b}$ ) can use to reach the destination.

To summarize, the search for new candidate abstractions involves (1) splitting nodes in the initial abstraction, (2) repairing the abstraction to ensure the forallexists condition holds, (3) checking that the generated abstraction is plausible, and if not, (4) splitting additional nodes on the min cut. This iterative process will often generate many candidates. The breadth parameter of the search bounds the total number of plausible candidates we will generate in between verification efforts. Of all the plausible candidates generated, we choose the smallest one to verify using the SMT solver.

\subsection{Learning from Counterexamples}

Any nodes of an abstraction that have a min cut of less than $k+1$ definitely cannot tolerate $k$ faults. If an abstraction is plausible, it satisfies a necessary condition for source-destination connectivity, but not a sufficient one - misconfigured routing policy can still cause nodes to be unreachable by modifying and/or subsequently dropping routing messages. For instance, the abstract network of Fig. 3c 
is plausible for one failure, but if $\widehat{b}$ 's routing policy blocks routes of either $\widehat{a}_{13}$ or $\widehat{a}_{24}$ then the abstract network will not be 1 -fault tolerant. Indeed, it is the complexity of routing policy that necessitates a heavy-weight verification procedure in the first place, rather than a simpler graph algorithm alone.

In a plausible abstraction, if the verifier computes a solution to the network that violates the desired fault-tolerance property, some node could not reach the destination because one or more of their paths to the destination could not be used to route traffic. We use the generated counterexample to learn edges that could not be used to route traffic due to the policy on them. To do so, we inspect the computed solution to find nodes $\widehat{u}$ that (1) lack a route to the destination (i.e. $\widehat{\mathcal{L}}(\widehat{u})=\infty),(2)$ have a neighbor $\widehat{v}$ that has a valid route to the destination, and (3) the link between $\widehat{u}$ and $\widehat{v}$ is not failed. These conditions imply the absence of a valid route to the destination not because link failures disabled all paths to the destination, but because the network policy dropped some routes. For example, in picture Fig. $3 c$, consider the case where $\widehat{b}$ does not advertise routes from $\widehat{a}_{13}$ and $\widehat{a}_{24}$; if the link between $\widehat{a}_{13}$ and $\widehat{d}$ fails, then $\widehat{a}_{13}$ has no route the destination and we learn that the edge $\left\langle\widehat{b}, \widehat{a}_{13}\right\rangle$ cannot be used. In fact, since $\widehat{a}_{13}$ and $\widehat{a}_{12}$ belonged to the same abstract group $\widehat{a}$ before we split them, their routing policies are equal modulo the abstraction function by trans-equivalence. Hence, we can infer that in a symmetric scenario, the link $\left\langle\widehat{b}, \widehat{a}_{24}\right\rangle$ will also be unusable.

Given a set of unuseable edges, learned from a counterexample, we restrict the min cut problems that define the plausible abstractions, by disallowing the use of those edges. Essentially, we enrich the refinement algorithm's topological based analysis (based on min-cut) with knowledge about the policy; the algorithm will have to generate abstractions that are plausible without using those edges. With those edges disabled, the refinement process continues as before.

\section{Implementation}

Origami uses the Batfish network analysis framework [12] to parse network configurations, and then translate them into a pure functional intermediate representation (IR) designed for network verification. This IR represents the structure of routing messages and the semantics of transfer and preference relations using standard functional data structures.

The translation generates a separate functional program for each destination subnet. In other words, if a network has 100 top-of-rack switches and each such switch announces the subnets for 30 adjacent hosts, then Origami generates 100 functional programs (i.e. problem instances). We separately apply our algorithms to each problem instance, converting the functional program to an SMT formula when necessary according to the algorithm described earlier. Since vendor routing configuration languages have limited expressive power (e.g., no loops or recursion) the translation requires no user-provided invariants. We use Z3 [10] to determine satisfiability of the SMT problems. Solving the problems separately (and in parallel) provides a speedup over solving the routing problem for all destinations simultaneously: The individual problems are specialized to a 
particular destination. By doing so, opportunities for optimizations that reduce the problem size, such as dead code elimination, arise.

Optimizing Refinement: During the course of implementing Origami, we discovered a number of optimizations to the refinement phase.

- If the min-cut between the destination and a vertex $u$ is less than or equal to the desired number of disjoint paths, then we do not need to compute another min-cut for the nodes in the unreachable portion of vertices $T$; we know nodes in $T$ can be disconnected from the destination. This significantly reduces the number of min-cut computations.

- We stop exploring abstractions that are larger in size than the smallest plausible abstraction computed since the last invocation of the SMT solver.

- We bias our refinement process to explore the smallest abstractions first. When combined the previous optimization, this prunes our search space from some abstractions that were unnecessary large.

Minimizing Counterexamples: When the SMT solver returns a counterexample, it often uses the maximum number of failures. This is not surprising as maximizing failures simplifies the SMT problem. Unfortunately, it also confounds our analysis to determine whether a counterexample is real or spurious.

\begin{tabular}{|c|c|c|c|c|c|c|c|}
\hline Topo & Con V/E & Fail & Abs V/E & Ratio & Abs Time & SMT Calls & SMT Time \\
\hline \multirow{4}{*}{ FT20 } & \multirow{4}{*}{$500 / 8000$} & 1 & $9 / 20$ & $55.5 / 400$ & 0.1 & 1 & 0.1 \\
\hline & & 3 & $40 / 192$ & $12.5 / 41.67$ & 1.0 & 2 & 7.6 \\
\hline & & 5 & $96 / 720$ & $5.20 / 11.1$ & 2.5 & 2 & 248 \\
\hline & & 10 & $59 / 440$ & $8.48 / 18.18$ & 0.9 & - & - \\
\hline \multirow{3}{*}{ FT40 } & \multirow{3}{*}{$2000 / 64000$} & 1 & $12 / 28$ & $166.7 / 2285.7$ & 0.1 & 1 & 0.1 \\
\hline & & 3 & $45 / 220$ & $44.4 / 290.9$ & 33 & 2 & 12.3 \\
\hline & & 5 & $109 / 880$ & $18.34 / 72.72$ & 762.3 & 2 & 184.1 \\
\hline \multirow{3}{*}{ SP40 } & \multirow{3}{*}{$2000 / 64000$} & 1 & $13 / 32$ & $153.8 / 2000$ & 0.2 & 1 & 0.1 \\
\hline & & 3 & $39 / 176$ & $51.3 / 363.6$ & 30.3 & 1 & 2 \\
\hline & & 5 & $79 / 522$ & $25.3 / 122.6$ & 372.2 & 1 & 22 \\
\hline \multirow{3}{*}{ FbFT } & \multirow{3}{*}{$744 / 10880$} & 1 & $20 / 66$ & $37.2 / 164.8$ & 0.1 & 3 & 1 \\
\hline & & 3 & $57 / 360$ & $13.05 / 30.22$ & 1 & 4 & 18.3 \\
\hline & & 5 & $93 / 684$ & $8 / 15.9$ & 408.9 & - & - \\
\hline
\end{tabular}

Fig. 4. Compression results. Topo: the network topology. Con V/E: Number of nodes/edges of concrete network. Fail: Number of failures. Abs V/E: Number of nodes/edges of the best abstraction. Ratio: Compression ratio (nodes/edges). Abs Time: Time taken to find abstractions (sec.). SMT Calls: Number of calls to the SMT solver. SMT Time: Time taken by the SMT solver (sec.).

To mitigate the effect of this problem, we could ask the solver to minimize the returned counterexample, returning a counterexample that corresponds to the fewest concrete link failures. We could do so by providing the solver with additional constraints specifying the number of concrete links that correspond 
to each abstract link and then asking the solver to return a counterexample that minimizes this sum of concrete failures. Of course, doing so requires we solve a more expensive optimization problem. Instead, given an initial (possibly spurious counter-example), we simple ask the solver to find a new counterexample that (additionally) satisfies this constraint. If it succeeds, we have found a real counterexample. If it fails, we use it to refine our abstraction.

\section{Evaluation}

We evaluate Origami on a collection of synthetic data center networks that are using BGP to implement shortest-paths routing policies over common industrial datacenter topologies. Data centers are good fit for our algorithms as they can be very large but are highly symmetrical and designed for fault tolerance. Data center topologies (often called fattree topologies) are typically organized in layers, with each layer containing many routers. Each router in a layer is connected to a number of routers in the layer above (and below) it. The precise number of neighbors to which a router is connected, and the pattern of said connections, is part of the topology definition. We focus on two common topologies: fattree topologies used at Google (labelled FT20, FT40 and SP40 below) and a different fattree used at Facebook (labelled FB12). These are relatively large data center topologies ranging from 500 to 2000 nodes and 8000 to 64000 edges.

SP40 uses a pure shortest paths routing policy. For other experiments (FT20, FT40, FB12), we augment shortest paths with additional policy that selectively drops routing announcements, for example disabling "valley routing" in various places which allows up-down-up-down routes through the data centers instead of just up-down routes. The pure shortest paths policy represents a best-case scenario for our technology as it gives rise to perfect symmetry and makes our heuristics especially effective. By adding variations in routing policy, we provide a greater challenge for our tool.

Experiments were done on a Mac with a $4 \mathrm{GHz}$ i7 $\mathrm{CPU}$ and $16 \mathrm{~GB}$ memory.

\subsection{Compression Results}

Figure 4 shows the level of compression achieved, along with the required time for compression and verification. In most cases, we achieve a high compression ratio especially in terms of links. This drastically reduces the possible failure combinations for the underlying verification process. The cases of 10 link failures on FT20 and 5 link failures on FbFT demonstrate another aspect of our algorithm. Both topologies cannot sustain that many link failures, i.e. some concrete nodes have less than 10 (resp. 5) neighbors. We can determine this as we refine the abstraction; there are (abstract) nodes that do not satisfy the min cut requirement and we cannot refine them further. This constitutes an actual counterexample and explains why the abstraction of FT20 for 10 link failures is smaller than the one for 5 link failures. Importantly, we did not use the SMT solver to find this counterexample. Likewise, we did not need to run a min cut on 
the much larger concrete topology. Intuitively, the rest of the network remained abstract, while the part that led to the counterexample became fully concrete.

\subsection{Verification Performance}

The verification time of Origami is dominated by abstraction time and SMT time, which can be seen in Fig. 4. In practice, there is also some time taken to parse and pre-process the configurations but it is negligible. The abstraction time is highly dependent on the size of the network and the abstraction search breadth used. In this case, the breadth was set to 25 , a relatively high value.

While the verification time for a high number of link failures is not negligible, we found that verification without abstraction is essentially impossible. We used Minesweeper [2], the state-of-the-art SMT-based network verifier, to verify the same fault tolerance properties and it was unable to solve any of our queries. This is not surprising, as SMT-based verifiers do not scale to networks beyond the size of FT20 even without any link failures.

\subsection{Refinement Effectiveness}

We now evaluate the effectiveness of our search and refinement techniques.

Effectiveness of Search. To assess the effectiveness of the search procedure, we compute an initial abstraction of the FT20 network suitable for 5 link failures, using different values of the search breadth. On top of this, we additionally consider the impact of some of the heuristics described in Sect. 5 . Figure 5 presents the size (the number of nodes are on the $y$ axis and the number of edges on top of the bars) of the computed abstractions with respect to various values for the breadth of search and sets of heuristics:

- Heuristics off means that (almost) all heuristics are turned off. We still try to split nodes that are on the cut-set.

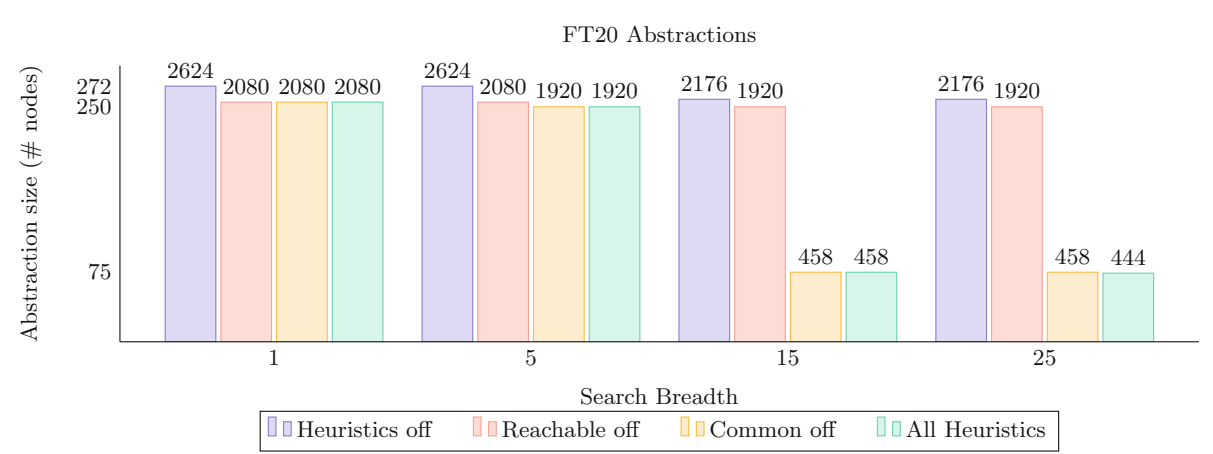

Fig. 5. The initial abstraction of FT20 for 5 link failures using different heuristics and search breadth. On top of the bars is the number of edges of each abstraction. 
- Reachable off means that we do not bias towards splitting of nodes in the reachable portion of the cut-set.

- Common off means that we do not bias towards splitting reachable nodes that have the most connections to unreachable nodes.

The results of this experiment show that in order to achieve effective compression ratios we need to employ both smart heuristics and a wide search through the space of abstractions. It is possible that increasing the search breadth would make the heuristics redundant, however, in most cases this would make the refinement process exceed acceptable time limits.

Use of Counterexamples. We now assess how important it is to (1) use symmetries in policy to infer more information from counterexamples, and (2) minimize the counterexample provided by the solver.

We see in Fig. 6 that disabling them increases number of refinement iterations. While each of these refinements is performed quickly, the same cannot be guaranteed of the verification process that runs between them. Hence, it is important to keep refinement iterations as low as possible.

\section{Related Work}

Our approach to network fault-tolerance verification draws heavily from ideas in prior work exploiting symmetry and abstraction in model checking $[4,6,17]$ and automatic abstraction refinement via CEGAR $[1,5,9]$. However, we apply these ideas to network routing, which introduces different challenges and opportunities. For example, our notion of abstraction ( $\forall \exists$-abstraction) differs from the typical existential abstraction used in model checking [6]. In addition, we have to deal with network topological structure and asymmetries introduced by failures.

Bonsai [3] and Surgeries [22] both leverage abstraction to accelerate verification for routing protocols and packet forwarding respectively. Both tools compute a single abstract network that is bisimilar to the original concrete network. Alas, neither approach can be used to reason about properties when faults may occur.

Minesweeper [2] is a general approach to control plane verification based on a stable state encoding, which leverages an SMT solver in the back-end. It supports a wide range of routing protocols and properties, including fault tolerance properties. Our compression is complementary

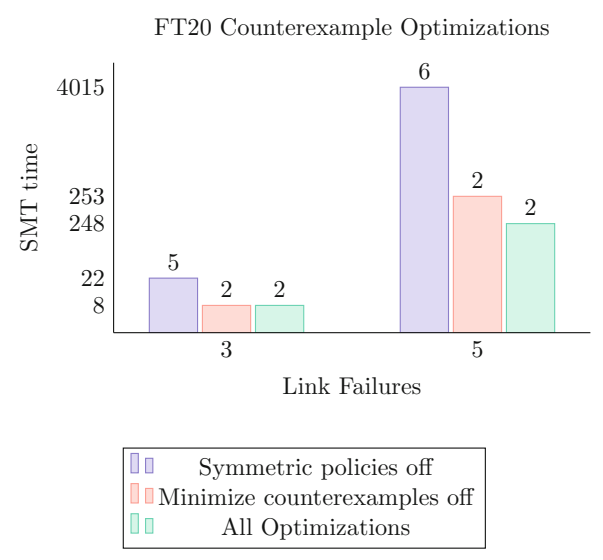

Fig. 6. Effectiveness of minimizing counterexamples and of learning unused edges. On top of the bars is the number of SMT calls. 
to such tools; it is used to alleviate the scaling problem that Minesweeper faces with large networks.

With respect to verification of fault tolerance, ARC [13] translates a limited class of routing policies to a weighted graph where fault-tolerance properties can be checked using graph algorithms. However, ARC only handles shortest path routing and cannot support stateful features such as BGP communities, or local preference, etc. While ARC applies graph algorithms on a statically-computed graph, we use graph algorithms as part of a refinement loop in conjunction with a general purpose solver.

\section{Conclusions}

We present a new theory of distributed routing protocols in the presence of bounded link failures, and we use the theory to develop algorithms for network compression and counterexample-guided verification of fault tolerance properties. In doing so, we observe that (1) even though abstract networks route differently from concrete ones in the presence of failures, the concrete routes wind up being "at least as good" as the abstract ones when networks satisfy reasonable well-formedness constraints, and (2) using efficient graph algorithms (min cut) in the middle of the CEGAR loop speeds the search for refinements.

We implemented our algorithms in a network verification tool called Origami. Evaluation of the tool on synthetic networks shows that our algorithms accelerate verification of fault tolerance properties significantly, making it possible to verify networks out of reach of other state-of-the-art tools.

\section{References}

1. Ball, T., Majumdar, R., Millstein, T.D., Rajamani, S.K.: Automatic predicate abstraction of $\mathrm{C}$ programs. In: Proceedings of the 2001 ACM SIGPLAN Conference on Programming Language Design and Implementation (PLDI), pp. 203-213 (2001)

2. Beckett, R., Gupta, A., Mahajan, R., Walker, D.: A general approach to network configuration verification. In: SIGCOMM, August 2017

3. Beckett, R., Gupta, A., Mahajan, R., Walker, D.: Control plane compression. In: Proceedings of the 2018 Conference of the ACM Special Interest Group on Data Communication, pp. 476-489. ACM (2018)

4. Clarke, E.M., Filkorn, T., Jha, S.: Exploiting symmetry in temporal logic model checking. In: Courcoubetis, C. (ed.) CAV 1993. LNCS, vol. 697, pp. 450-462. Springer, Heidelberg (1993). https://doi.org/10.1007/3-540-56922-7_37

5. Clarke, E., Grumberg, O., Jha, S., Lu, Y., Veith, H.: Counterexample-guided abstraction refinement. In: Emerson, E.A., Sistla, A.P. (eds.) CAV 2000. LNCS, vol. 1855, pp. 154-169. Springer, Heidelberg (2000). https://doi.org/10.1007/ 10722167_15

6. Clarke, E.M., Grumberg, O., Long, D.E.: Model checking and abstraction. ACM Trans. Program. Lang. Syst. 16(5), 1512-1542 (1994)

7. Daggitt, M.L., Gurney, A.J.T., Griffin, T.G.: Asynchronous convergence of policyrich distributed bellman-ford routing protocols. In: SIGCOMM, pp. 103-116 (2018) 
8. Daggitt, M.L., Gurney, A.J., Griffin, T.G.: Asynchronous convergence of policy-rich distributed Bellman-Ford routing protocols. In: Proceedings of the 2018 Conference of the ACM Special Interest Group on Data Communication, pp. 103-116. ACM (2018)

9. Das, S., Dill, D.L.: Successive approximation of abstract transition relations. In: Proceedings of the 16th Annual IEEE Symposium on Logic in Computer Science, LICS 2001, p. 51 (2001)

10. de Moura, L., Bjørner, N.: Z3: an efficient SMT solver. In: Ramakrishnan, C.R., Rehof, J. (eds.) TACAS 2008. LNCS, vol. 4963, pp. 337-340. Springer, Heidelberg (2008). https://doi.org/10.1007/978-3-540-78800-3_24

11. Feamster, N., Rexford, J.: Network-wide prediction of BGP routes. IEEE/ACM Trans. Netw. 15(2), 253-266 (2007)

12. Fogel, A., et al.: A general approach to network configuration analysis. In: NSDI (2015)

13. Gember-Jacobson, A., Viswanathan, R., Akella, A., Mahajan, R.: Fast control plane analysis using an abstract representation. In: SIGCOMM (2016)

14. Gill, P., Jain, N., Nagappan, N.: Understanding network failures in data centers: measurement, analysis, and implications. In: SIGCOMM (2011)

15. Godfrey, J.: The summer of network misconfigurations (2016). https://blog. algosec.com/2016/08/business-outages-caused-misconfigurations-headline-newssummer.html

16. Griffin, T.G., Shepherd, F.B., Wilfong, G.: The stable paths problem and interdomain routing. IEEE/ACM Trans. Netw. 10(2), 232-243 (2002)

17. Kesten, Y., Pnueli, A.: Control and data abstraction: the cornerstones of practical formal verification. Softw. Tools Technol. Transf. 2(4), 328-342 (2000)

18. Lapukhov, P., Premji, A., Mitchell, J.: Use of BGP for routing in large-scale data centers. Internet draft (2015)

19. Lopes, N.P., Rybalchenko, A.: Fast BGP simulation of large datacenters. In: Enea, C., Piskac, R. (eds.) VMCAI 2019. LNCS, vol. 11388, pp. 386-408. Springer, Cham (2019). https://doi.org/10.1007/978-3-030-11245-5_18

20. Lougheed, K.: A border gateway protocol (BGP). RFC 1163, RFC Editor (1989). http://www.rfc-editor.org/rfc/rfc1163.txt

21. Mahajan, R., Wetherall, D., Anderson, T.: Understanding BGP misconfiguration. In: SIGCOMM (2002)

22. Plotkin, G.D., Bjørner, N., Lopes, N.P., Rybalchenko, A., Varghese, G.: Scaling network verification using symmetry and surgery. In: POPL (2016)

23. Quoitin, B., Uhlig, S.: Modeling the routing of an autonomous system with C-BGP. Netw. Mag. Glob. Internetworking 19(6), 12-19 (2005)

24. Sobrinho, J.A.L.: An algebraic theory of dynamic network routing. IEEE/ACM Trans. Netw. 13(5), 1160-1173 (2005)

25. Weitz, K., Woos, D., Torlak, E., Ernst, M.D., Krishnamurthy, A., Tatlock, Z.: Formal semantics and automated verification for the border gateway protocol. In: NetPL (2016) 
Open Access This chapter is licensed under the terms of the Creative Commons Attribution 4.0 International License (http://creativecommons.org/licenses/by/4.0/), which permits use, sharing, adaptation, distribution and reproduction in any medium or format, as long as you give appropriate credit to the original author(s) and the source, provide a link to the Creative Commons license and indicate if changes were made.

The images or other third party material in this chapter are included in the chapter's Creative Commons license, unless indicated otherwise in a credit line to the material. If material is not included in the chapter's Creative Commons license and your intended use is not permitted by statutory regulation or exceeds the permitted use, you will need to obtain permission directly from the copyright holder. 